\title{
Wheeled Mobile Robot Obstacle Avoidance Using Compass and Ultrasonic
}

\author{
Yousef Moh. Abueejela*, Haithem A. Ali \\ Control Engineering Department, College of Electronic Technology, Bani Walid, Libya
}

Copyright@2018 by authors, all rights reserved. Authors agree that this article remains permanently open access under the terms of the Creative Commons Attribution License 4.0 International License

\begin{abstract}
This paper presents the use of ARDUINO board to control an autonomous mobile robot (AMR) for navigation purpose. The wheeled robot is capable to perform two tasks. The first task is to move autonomously to the north direction. And the second task is to avoid collision with unexpected static and moveable obstacles. The robot uses two sensors to navigate and avoid the obstacle, a digital compass HMC5883L uses to detect the north direction and update the situation of the robot during its movement. And the ultrasonic sensor uses to avoid nearest obstacles on the robot way. C language used to program the system to do its mission and installed to the ARDUINO board. The results obtained show that the robot was able to navigate and move to the north and avoid obstacles in the outdoor environment.
\end{abstract}

Keywords Digital Compass Sensor HMC5883L, Ultrasonic Sensor, Autonomous Robot, Avoidance, Arduino, Navigation

\section{Introduction}

An autonomous mobile robot (AMR) is defined as a wheeled vehicle that capable of moving in an environment autonomously. Autonomous is the ability of moving and navigate without assistance from external operator, with the availability of internal or external sensors that get information of the environment.
The most common external sensors are (ultrasonic, laser, infrared, etc.) to detect obstacles and measure the distance between the obstacle and the robot. The AMR is equipped with a set of motorized actuators and some of sensors to help it to move and navigate. In order to control its motion, usually, there is an on-board computer to command the motors to take place, based on reference inputs and the data gathered by the sensors.

When autonomous robots navigate within outdoor environments (open space), they have to be endowed the ability to move through the environments, to move without collision with obstacles, and follow the direction with the help of (compass, GPS or camera). The basic concept of this project report is to design robot which can move to the north direction and avoid any obstacle on its way without human guidance or control. Arduino Mega 2560 was used as the "brain" to control the system of the robot $[1-3,6]$. Figure 1 shows the prototype system.

The robot composes the following sensors and actuators:

- One ultrasonic range finder mounted in front of the mobile robot to detect obstacles and provide information to the controller.

- One servomotor attached to the ultrasonic to rotate the ultrasonic from 0 degree to 180 degree.

- Digital compass sensor type HMC5883L to provide information about the direction of the robot to the controller.

- An infra-red to switch ON/OFF the robot remotely. 


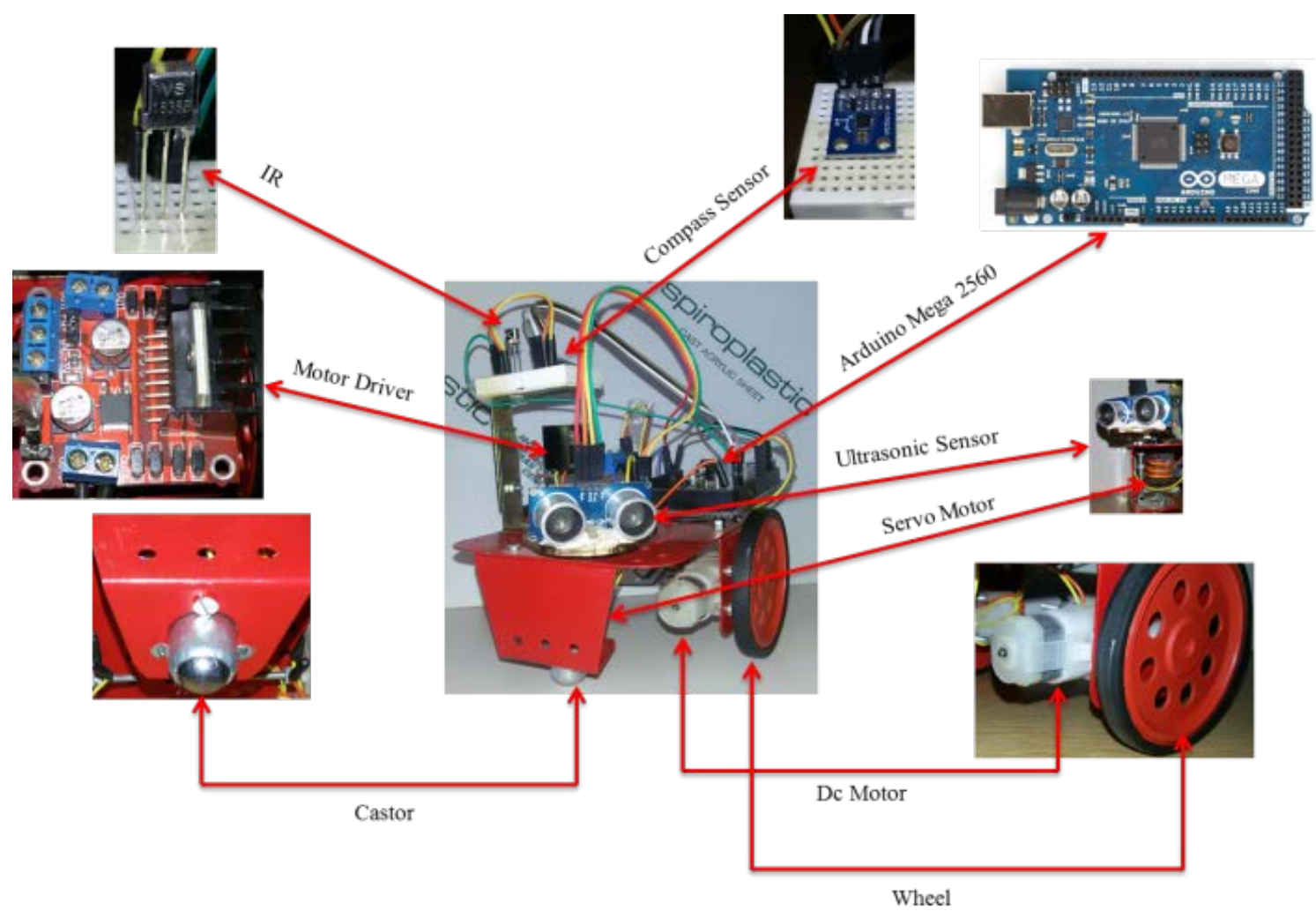

Figure 1. System Prototype of the WMR

\section{Problem Statement}

One of the wheeled mobile robot problems in outdoor environment is how to choose its path to move from point to point, and how can get the capability to avoid unexpected obstacles around or on its way. Therefore, one of the solutions proposed for this issue is presented by choosing ultrasonic sensor and digital compass sensor to give the robot the ability to move freely in unknown environment. The compass sensor is used to detect the path for the robot, and the ultrasonic sensor is used to help the robot to avoid any obstacle in front the robot while moving.

\section{Methodology}

The wheeled mobile robot prototype is divided into two main stages which are the hardware and software. The hardware consists many parts (chassis, wheels, DC motors, sensors and on board controller). Each part tested in real time and implemented separately, and then integrated together to construct the robot system. Software part was done by using C language with ARDUINO interface board. In this section, input devices, output devices and mechanical structure will be discussed and introduce.

\subsection{System Block Diagram}

Figure 2 shows the complete system diagram of wheeled mobile robot and its controller board with two inputs (ultrasonic and digital compass). The ARDUINO controller was used as the main brain of the whole system; it receives the data from the input devices and updates the outputs devices. The target point for the system is the North Direction; so that the digital compass sensor used to feed back the controller with the mobile robot direction. Furthermore, ultrasonic sensors proposed to complete the navigation of robot driving to avoid the robot to hit the walls or obstacles.

The prototype robot consists of a combination of electronic, electrical and mechanical parts, the whole system proposed are:

- Input Devices are used to start, gather information about the system and the outdoor environment, which consists switch (remote control IR), sensors (ultrasonic sensor and Digital Compass), in order to feed the controller (Microcontroller) by information about the surrounding environment to take the decision.

- The ARDUINO board is an integrated circuit that contains a processor, memory and a programmable input and output pins. The controller is the brain of the robot, stores and sends programmed tasks to other parts of the main robot.

- Output Devices are the actuators that convert an electrical signal into mechanical movement; the types of actuators are:

Servo motor to rotate the ultrasonic sensor from $(0-180$ degree), and 


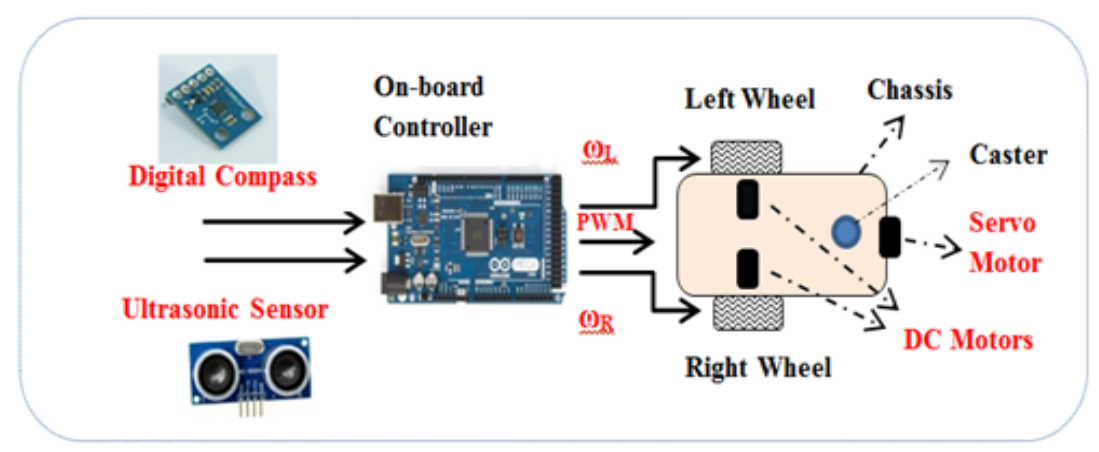

Figure 2. System Architecture of the WMR

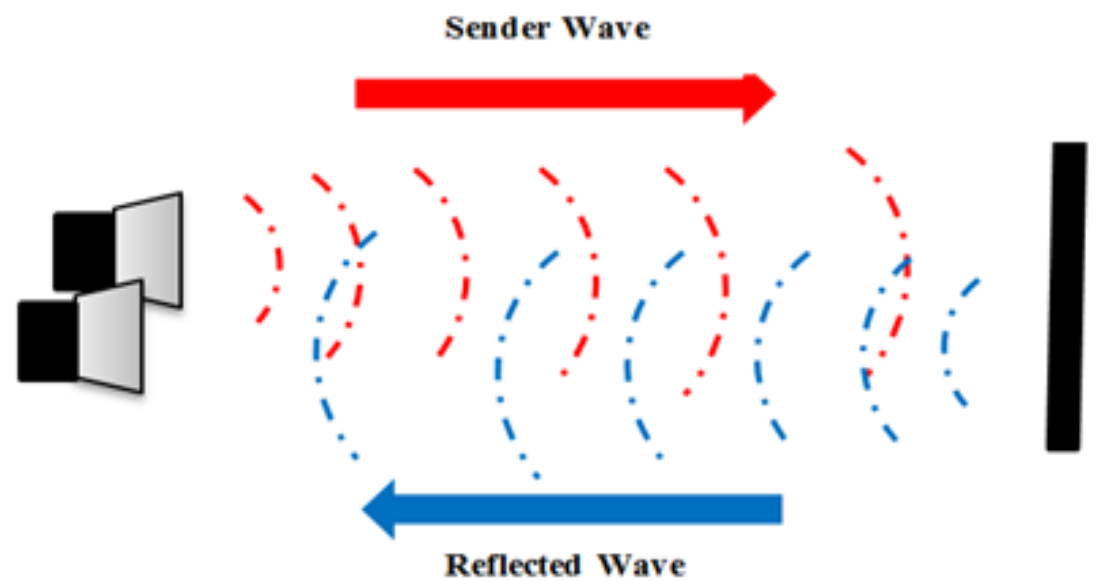

Figure 3. Ultrasonic Transmission/Reflecting signal with an Object

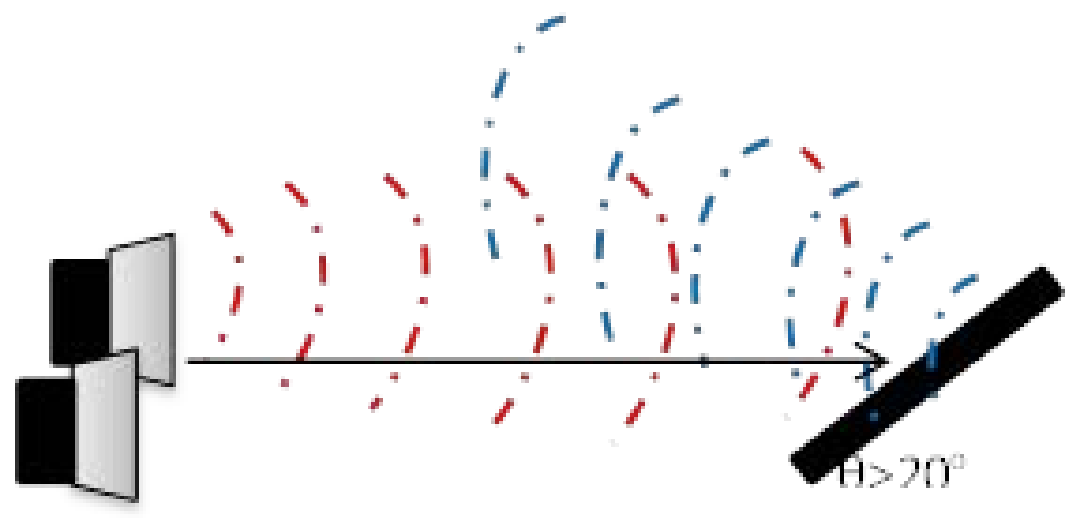

Figure 4. Ultrasonic Transmission/Reflecting signal with an Object

- Two DC motors attached with two wheels to drive the robot.

\subsection{Ultrasonic Sensor and Limitation}

An ultrasonic sensor HC - SR04 transmits ultrasonic waves into the air and detects reflected waves from an object as shown in Figure 3, ultrasonic provides $2 \mathrm{~cm}-$ $400 \mathrm{~cm}$ non-contact measurement function. The distance between the robot and the obstacle is determined by

$$
d=\frac{v t}{2}
$$

Where:

$d$ : is the distance measured. $t$ : is the time traveling from the sensor the object. $v$ : is the sound velocity on air.

Ultrasonic measurement has some limitations; in case of the surface is perpendicular to the ultrasonic sensor, the ultrasonic waves are reflected and will be detected by receiver then the object can be selected as shown in Figure3, while only a small percentage of the waves is scattered in other directions. However, if the surface is tilted by an large angel as shown in Figure 4 , then the obstacle will not been detected $[4,5,10]$.

The surface structure of the obstacle effects on the amount of reflected waves. Furthermore, the size of the irregularities on the surface should be comparable to the 
wavelength of the incident waves [5].

$$
\lambda=\frac{v}{f}=\frac{340}{40000}=8.5 \mathrm{~mm}
$$

Where

$\lambda$ is the wavelength,

$\mathrm{v}$ is sound velocity in air ( $340 \mathrm{~m} / \mathrm{s})$,

$f$ is ultrasonic sound frequency $40 \mathrm{KHz}$

Another problem arises when the angle $(\theta)$ is greater than 15 degree from the perpendicular line of sensor' sender, in this case the object may not detect by the robot. The problem can be minimizing by using servomotor drive to rotate the sensor by extra degrees while the robot move.

\subsection{Digital Compass and Limitations}

Digital compass is a surface-mount, multi-chip module designed for low field magnetic sensing with a digital interface. The HMC5883L includes 12-bit ADC that enables $1^{\circ}$ to $2^{\circ}$ compass heading accuracy.

Unfortunately, the digital compass affect by the ultrasonic waves, so that, we found the best way to avoid that is to make the two sensors work in sequence with delay 2 seconds. Another limitation of the compass sensor is affected by the magnetic fields when the robot approaching magnetic field or be above the dc motors.

\subsection{Kinematics Model of the Mobile Robot}

Kinematics is the study of the mathematics of motion without considering the forces that affect the motion. It deals with the geometric relationships that govern the system. It develops a relationship between control parameters and the behavior of a system in space $[3,7,8]$. The model of the robot is as shown in Figure5. The linear speed of the right and the left wheel $\left(v_{r}, v_{l}\right)$ are related to the angular speed as follows:

$$
\begin{aligned}
& v_{r}=r \omega_{r} \\
& v_{l}=r \omega_{l}
\end{aligned}
$$

Where:

$r$ : is the radius of the wheel.

$\omega_{\mathrm{r}}$ and $\omega_{\mathrm{r}}$ : angular velocity of the right and left wheels.
The total linear and angular speed of the robot can be calculated as equations (5) and (6). The robot in a state of motion must always rotate about a point that lies somewhere on the common axis of its two wheels. This point is often called the Instantaneous Centre of Curvature (ICC). In order to derive the differential drive equations, the model shown in Figure 5 is used.

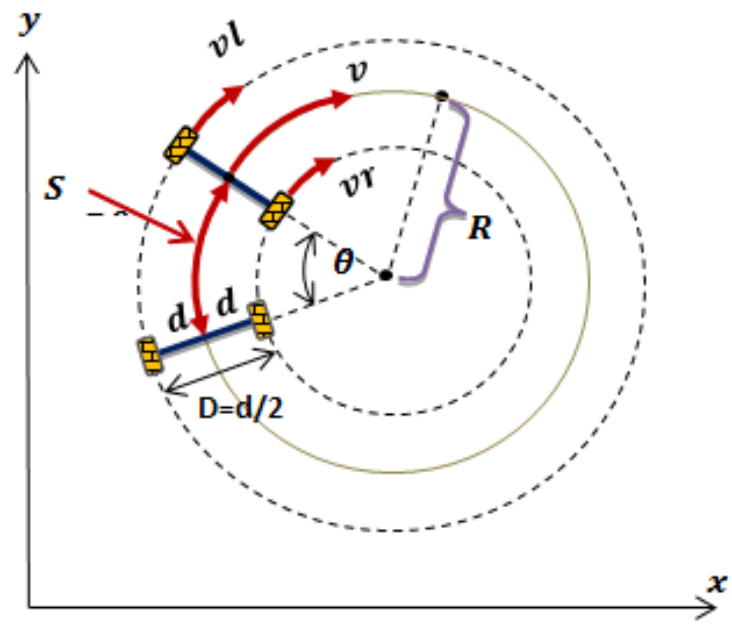

Figure 5. The representation of a wheeled mobile robot

$$
\begin{gathered}
v=\frac{v_{r}(\mathrm{t})+v_{l}(\mathrm{t})}{2} \\
R=\frac{v(t)}{\omega(t)}=\frac{D}{2} \frac{\left(v_{r}(t)+v_{l}(t)\right)}{\left(v_{r}(t)-v_{l}(t)\right)}
\end{gathered}
$$

Where:

$v, \omega$ : is the velocity and angular velocity of the robot

$R$ : instantaneous radius of curvature (ICC).

$\mathrm{S}$ : is the robot curvature travel.

There are three cases of the robot motion we could define, as shown in Figure 6.

If $v_{l}=v_{r}$, then we have forward linear motion in a straight line. $\mathrm{R}$ becomes infinite, and there is effectively no rotation $\theta$ is zero $[3,9]$.

If $v_{l}>v_{r}$, then we have right rotation with $\mathrm{R}$

If $v_{l}<v_{r}$, then we have left rotation with $\mathrm{R}$.

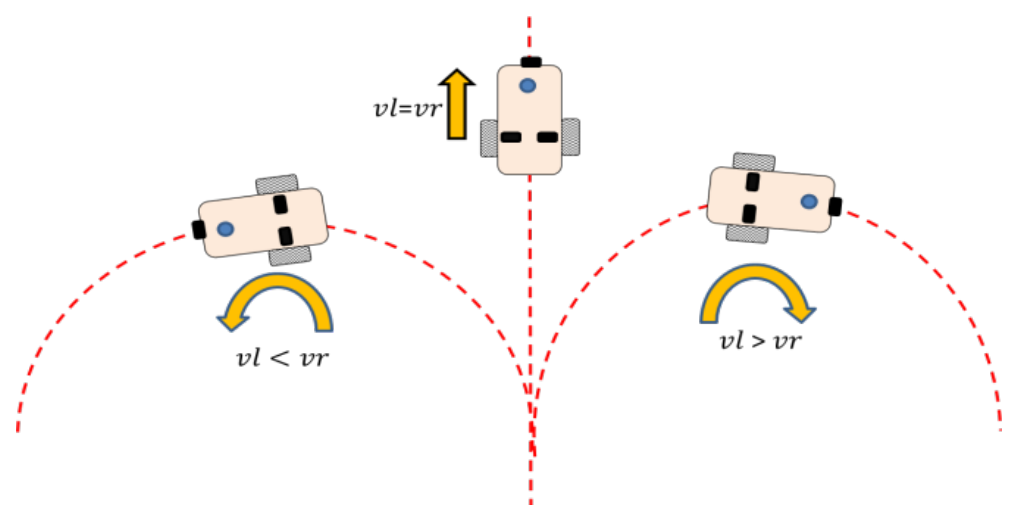

Figure 6. Robot cases of rotations 


\section{Experimental Results}

In this section, we present experiments results and algorithm illustration of the presented techniques.When the system starts, servo motor rotates the ultrasonic sensor with five corners as shown in Figure 8, and at every angle the robot calculate the distance of nearest obstacle, and then compares the distances to know where the obstacles are, after that the robot getting its direction by using compass sensor and looking for the desired path by calculating the angle of steering, Figure 7 shows the flowchart of the process.

\subsection{Testing the Ultrasonic Distance Sensor}

To get accurate distance measurement, we send a very short HIGH signal of 10 microseconds $(\mu \mathrm{S})$ to the Trigg pin. After 3 seconds, the sensor should return a HIGH signal again to measure another value. We found that, this procedure was done to avoid an unstable measuring duo to multi reflected waves from the surrounding, and we get a perfect stable value which is $15 \mathrm{~cm}$.

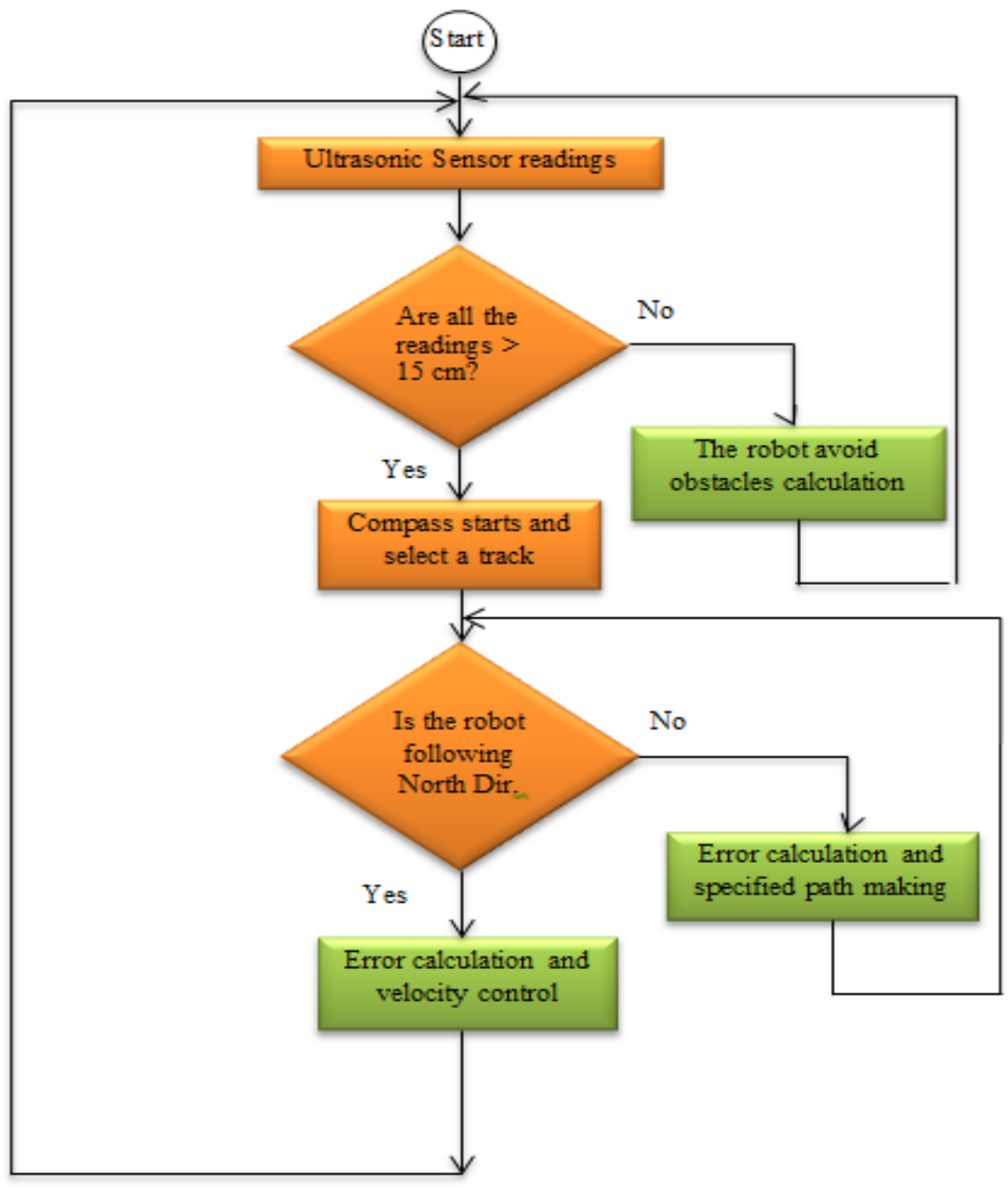

Figure 7. Flowchart of wheeled mobile robot
40
60
90
120
160

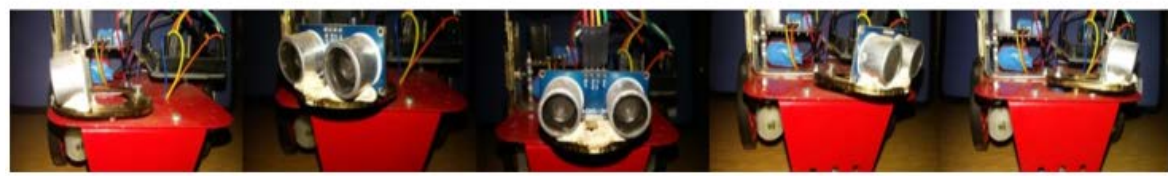

Figure 8. Ultrasonic mounted with the Servo motor 


\subsection{Testing the Digital Compass Sensor}

During the experiments and results, we found that the digital compass must be lie far from the DC motors and avoid activate it with ultrasonic sensor at the same time. So that, after the ultrasonic deactivate we should wait 1 second and then start reading compass data.

\subsection{System Implementation and Running}

After the operation of the system, the robot scans the surrounding environment to detect nearest obstacle if the obstacle distance is greater than $15 \mathrm{~cm}$ the robot will move forward. Otherwise the robot will take a decision to true left or right as shown in Figure 9 to avoid the obstacle and search again for the North direction.
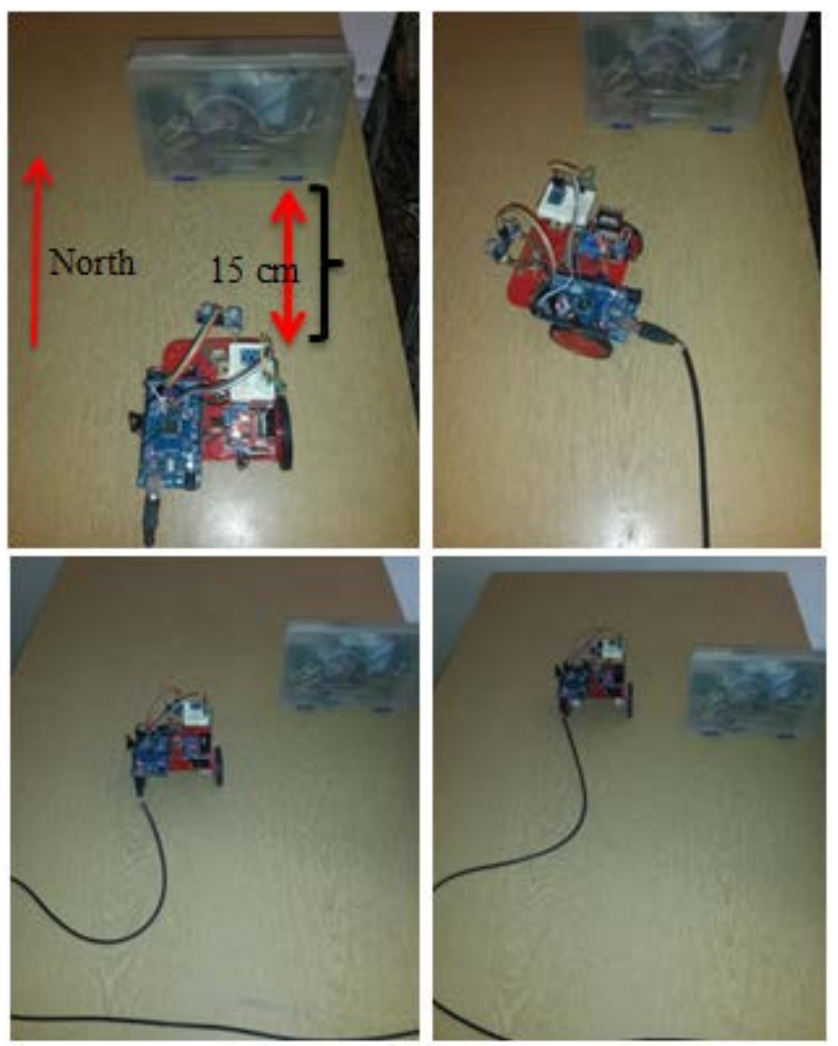

Figure 9. Robot motion

\section{Technical Specification}

Table 1. Technical specifications of the prototype

\begin{tabular}{|c|c|}
\hline Item & Description \\
\hline Robot Size & $11.5 \mathrm{~cm}(\mathrm{~L}) \times 10.5 \mathrm{~cm}(\mathrm{~W}) \times 8.5 \mathrm{~cm}(\mathrm{H})$ \\
\hline Robot Weight & $0.48 \mathrm{Kg}$ \\
\hline Robot Power & $12 \mathrm{volt}$ \\
\hline Max Speed & $7 \mathrm{~cm} / \mathrm{s}$ \\
\hline No. of Wheels & 2 with one caster at front \\
\hline Wheel Radius & $3.5 \mathrm{~cm}$ \\
\hline No. of Sensors & 2 \\
\hline Type of Sensor & Digital compass and ultrasonic \\
\hline Dc Motor velocity & $200 \mathrm{rad} / \mathrm{sec}$ \\
\hline
\end{tabular}

\section{Conclusions}

This paper discusses the implementation and designing of wheeled mobile robot for navigation and obstacle avoidance. The system proposed was successfully done for navigation and avoidance using digital compass sensor and ultrasonic sensor, these sensors and the effect of their limitations on the navigation and obstacle avoidance were discussed. The experimental results show that the outdoor autonomous mobile robot was able to realize the autonomous navigation and avoidance task.

\section{Acknowledgements}

This work has been supported by Control Engineering Department, College of Electronic Technology Bani Walid.

\section{REFERENCES}

[1] Chung L. C., Bohan W., Yong C. Huang, "Ultra Sonic Range Finding FOR Distance Measuring in Coal mining," IJRET of International Journal of Research in Engineering and Technology, 03, pp. 313-316, Nov 2013.

[2] Guan S., Youtong Z., Dongdong W., "Detection and Control of a Wheeled Mobile Robot Based on Magnetic Navigation,”, IEEE of International conference on mechatronics and automation, pp. 18-25 ,Feb 2013

[3] D. Hanafi, Youse Moh. Abueejela, Mohamad F. Zakaria, "Wall Follower Autonomous Robot Development Applying Fuzzy Incremental Controller,” Intelligent Control and Automation, 2013, 4, pp. 1497-150, Jul 2005.

[4] Pifu Z.,Jason G.,Evangelos E., Peter H., "Navigation with IMU/GPS/Digital Compass with Unscented Kalman Filter,”, IEEE of International conference on mechatronics and automation, pp. 18-25,Feb 2013.

[5] Johann B., Yoram K., "Obstacle Avoidance with Ultrasonic Sensor,” IEEE of robotics and automation, Vol. 4, No. 2, Aprill 1988.

[6] Wesam J. and Safa K.,” designed an autonomous wheeled mobile robot for wall follower.” B.Sc., Dept. Cont. Eng., Collage of Electronics Technology, Baniwaled, 2011.

[7] Xi Li, Byung-Jae Choi, "Design of Obstacle Avoidance System for Mobile Robot using Fuzzy Logic Systems," IJSH of International Journal of Smart Home, Vol. 7, No. 3, May, 2013.

[8] S.A.S.Yapa, and etc., "Autonomous Obstacle Avoiding Differential Wheel Driven Robot," SAITM Research Symposium on Engineering Advancements 2014.

[9] M. Nițulescu, "Theoretical Aspects in Wheeled Mobile Robot Control,” 2008 IEEE.

[10] Mohamed Abdellatif and Osama A. Montasser, "Using Ultrasonic Range Sensors to Control a Mobile Robot in Obstacle Avoidance Behavior.” Ain Shams University, Faculty of Engineering Cairo, Egypt 2010. 